\title{
Pacific
}

Journal of

Mathematics

\section{STABLE RELATIONS. II. CORONA SEMIPROJECTIVITY AND} DIMENSION-DROP $C^{*}$-ALGEBRAS

TERRY ATHERTON LORING 


\title{
STABLE RELATIONS II: CORONA SEMIPROJECTIVITY AND DIMENSION-DROP $C^{*}$-ALGEBRAS
}

\author{
TERRY A. LORING
}

\begin{abstract}
We prove that the relations in any presentation of the dimension-drop interval are stable, meaning there is a perturbation of all approximate representations into exact representations. The dimension-drop interval is the algebra of all $M_{n}$-valued continuous function on the interval that are zero at one end-point and scalar at the other. This has applications to $\bmod -p K$-theory, lifting problems and classification problems in $C^{*}$-algebras. For many applications, the perturbation must respect precise functorial conditions. To make this possible, we develop a matricial version of Kasparov's technical theorem.
\end{abstract}

\section{Introduction.}

Suppose $\mathcal{R}$ is a finite set of relations on a finite set $G$ of generators so that $C^{*}\langle G \mid \mathcal{R}\rangle$ is isomorphic to the dimension-drop interval

$$
\tilde{\mathbb{I}}_{n}=\{f \in C[0,1] \mid f(0), f(1) \in \mathbb{C} I\} .
$$

For simplicity, we assume the relations are of the form $p\left(g_{1}, \ldots, g_{n}\right)=0$ for some $*$-polynomial $p$. Weak stability means that an approximate representation $\left(x_{1}, \ldots, x_{n}\right)$, meaning an $n$-tuple of elements in a $C^{*}$-algebra $A$ such that each $p\left(x_{1}, \ldots, x_{n}\right)$ is close zero, can be perturbed slightly within $A$ to an actual representation $\left(\tilde{x}_{1}, \ldots, \tilde{x}_{n}\right)$. That this (and a little more) can be done was shown in [8], but only for one specific set of relations. The relations $\mathcal{R}$ are stable if the pertubation can be done so that whenever there is a $*$-homomorphism $\varphi: A \rightarrow B$ which sends $\left(x_{1}, \ldots, x_{n}\right)$ to an exact representation, then $\varphi\left(\tilde{x}_{j}\right)=\varphi\left(x_{j}\right)$.

There are several advantages to stability over weak stability. It is far more useful when dealing with extensions of $C^{*}$-algebras and it depends only on the universal $C^{*}$-algebra, not the choice of relations for that $C^{*}$-algebra. The reason for our focus on the dimension-drop interval is primarily that this is the most complicated building block used in the inductive limits, called $\mathrm{AD}$ algebras, that appeared in Elliott's first classification paper [7]. 
See [5] for an application of stable relations to the extension problem for AD algebras. See [4] for a discussion of the role of the dimension-drop interval in mod- $p$ K-theory. Our results will be stated in the more general context of dimension-drop graphs, but certainly the dimension-drop interval is the most important case.

In $\S 2$ we give a characterization, in terms of lifting properties, of the universal $C^{*}$-algebras for stable relations. Since this property, called semiprojectivity, depends only on the $C^{*}$-algebra, this frees us from having to specify generators and relations in many cases. We have a third, equivalent property involving corona algebras. This characterization formalizes some of the ideas used by Olsen and Pedersen [11] to show that nilpotents always lift.

For any $C^{*}$-algebra $A$ we let $M(A)$ denote the multiplier algebra of $A$ and $C(A)$ denote the corona algebra $M(A) / A$.

By a dimension-drop graph, we mean a $C^{*}$-algebra of the form

$$
\left\{f \in C\left(X, M_{n}\right) \mid f(v) \in \mathbb{C} I \text { for all vertices } v\right\}
$$

where $X$ is the underlying topological space for a graph and $n$ is a positive integer. We call this a dimension-drop interval in the special case where $X$ is the unit interval with 0 and 1 as vertices.

To handle these algebras we need several generalizations of Kasparov's Technical Theorem. The purpose of these results is to show that, inside of a corona algebra, one can find good substitutes for elements that would exist if only the corona algebra were a von Neumann algebra. For example, there is an acceptable substitute for the logarithm of a unitary with full spectrum. Also, if $M_{n}(A)$ sits inside the corona algebra, there are elements that function just like matrix units in the way they multiply against $M_{n}(A)$, even if $A$ is not unital but only $\sigma$-unital.

These technical lemmas are very similar to the second splitting lemma in BDF [3, Lemma 7.3]. The basic form of these results is to show that every $\varphi: A \rightarrow C(E)$ factors through some injection $A \rightarrow A_{1}$. In the BDF case, $A$ and $A_{1}$ are commutative and $C(E)$ is the Calkin algebra.

Once we have shown that a dimension-drop graph is universal for a stable set of relations, a host of perturbation, lifting and homotopy results follow regarding homomorphisms (and asymptotic morphisms) out of dimensiondrop $C^{*}$-algebras. For most of these we refer the reader to [8] but we will mention one of these, [8, Theorem 3.8]. If a separable $C^{*}$-algebra $A$ has the property that any finite set of its elements can be approximated by elements of a $C^{*}$-subalgebra isomorphic to a quotient of a dimension-drop graph, then $A$ is the inductive limit of dimension-drop graphs.

A $C^{*}$-algebra that will figure prominently in all this the cone $\mathrm{C} M_{n}=$ $M_{n}\left(C_{0}(0,1]\right)$. By [8, Theorem 4.9] we know that $\mathrm{C} M_{n}$ is projective. This is 
a very useful fact as there are many copies of $\mathrm{C} M_{n}$ inside of a dimension-drop graph.

The author is grateful to Gert Pedersen for discussions which lead to much simplified proofs in Section four.

\section{A characterization of stability.}

We begin with a characterization of projectivity in terms of corona algebras that is suggested by [11]. This then generalizes to give a characterization of semiprojectivity and of stability for relations. One consequence is that two finite sets of relations that determine isomorphic universal $C^{*}$-algebras are either both stable, or both not.

All our definitions are with respect to the full category of not-necessarilyunital $C^{*}$-algebras and $*$-homomorphisms.

Definition 2.1. A $C^{*}$-algebra $A$ is projective if, for every surjection $\pi: B \rightarrow C$ and every *-homomorphism $\varphi: A \rightarrow C$, there exists a *homomorphism $\bar{\varphi}: A \rightarrow B$ such that $\pi \circ \bar{\varphi}=\varphi$. We call $A$ corona projective if this holds only in the special case where $C=C(E)$ for some $\sigma$-unital $C^{*}$-algebra $E$.

Theorem 2.2. Let $A$ be a separable $C^{*}$-algebra. Then $A$ is projective if and only if $A$ is corona projective.

Proof. The forward implication is trivial. Suppose that $A$ is corona projective and that $\varphi: A \rightarrow C$ and a surjection $\pi: B \rightarrow C$ are given. Replacing $B$, if necessary, by the closed span of a lift of a dense sequence in $\varphi(A)$ reduces the problem to the case where $B$ is separable.

Let $I=\operatorname{ker}(\pi)$ and let $I^{\perp}$ denote the annihilator of $I$ in $B$. As $I \cap I^{\perp}=0$ and $I+I^{\perp}$ is an essential ideal in $B$, we have the following commutative diagram with the left square a pull-back.

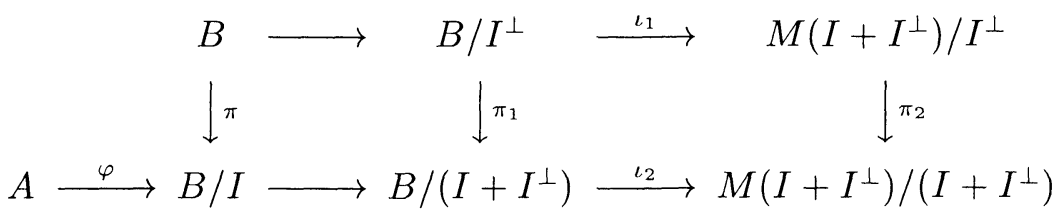

By the corona projectivity of $A$, we have

$$
\psi: A \rightarrow M\left(I+I^{\perp}\right) / I^{\perp}
$$

which is a lift of the composition of the bottom row:

We now claim that $\pi_{2}^{-1}\left(\operatorname{im}\left(\iota_{2}\right)\right) \subseteq \operatorname{im}\left(\iota_{1}\right)$. Suppose $b \in \pi_{2}^{-1}\left(\operatorname{im}\left(\iota_{2}\right)\right)$. Thus $\pi_{2}(b)=\iota_{2}(c)$ for some $c$. But $c=\pi_{1}(a)$ for some $a$, so

$$
\pi_{2}\left(\iota_{1}(a)\right)=\iota_{2}\left(\pi_{1}(a)\right)=\iota_{2}(c)=\pi_{2}(b) .
$$


This implies

$$
\iota_{1}(a)-b \in \operatorname{ker}\left(\pi_{2}\right)=\left(I+I^{\perp}\right) / I^{\perp} \subseteq B / I^{\perp}=\operatorname{im}\left(\iota_{1}\right)
$$

and hence $b \in \operatorname{im}\left(\iota_{1}\right)$.

By the claim, we may regard $\psi$ as a map into $B / I^{\perp}$. The pull-back property now shows that $\varphi$ and $\psi$ together determine the desired lifting to $B$.

Following Blackadar [1] we define semiprojectivity as a lifting property. This turns out to have better closure properties than the version of semiprojectivity due to Effros and Kaminker [6], which is better suited to some homotopy calculations.

Definition 2.3. A $C^{*}$-algebra $A$ is called semiprojective if, for every $*$-homomorphism $\varphi: A \rightarrow B / \overline{\cup I_{n}}$, where the $I_{n}$ are increasing ideals in $B$, and with $\pi_{m}: B / I_{m} \rightarrow B / \overline{\cup I_{n}}$ the natural quotient map, there exists, for some $m$, a *-homomorphism $\bar{\varphi}: A \rightarrow B / I_{m}$ such that $\pi_{m} \circ \bar{\varphi}=\varphi$. We call $A$ corona semiprojective if this holds only in the special case where $B / \overline{U I_{n}} \cong C(E)$ for some $\sigma$-unital $C^{*}$-algebra $E$.

Theorem 2.4. Let $A$ be a separable $C^{*}$-algebra. Then $A$ is semiprojective if and only if $A$ is corona semiprojective.

Proof. The proof is similar to that of Theorem 2.2 except that one uses the following diagram, with $I=\overline{U I_{n}}$.

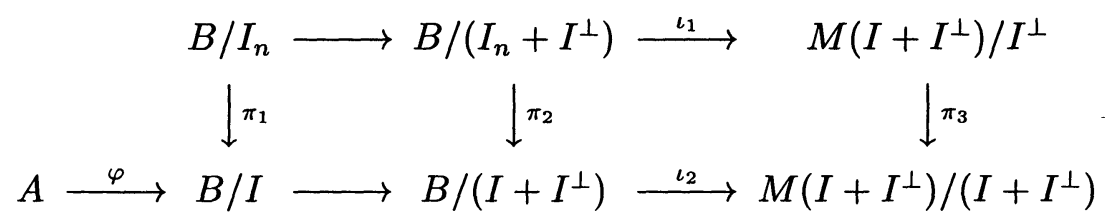

Notice that $\overline{U I_{n}+I^{\perp}}=I+I^{\perp}$, so corona semiprojectivity applies, and the left square is still a pull-back since $I \cap\left(I_{n}+I^{\perp}\right)=I_{n}$.

If $A$ is unital, then it is easy to see that one need only check the corona semiprojectivity condition in the special case $\varphi(1)=1$.

We now recall the definition of stability from [8]. We shall assume that $G=\left\{g_{1}, \ldots, g_{l}\right\}$ is a finite set of generators and $\mathcal{R}=\left\{p_{1}, \ldots, p_{k}\right\}$ is a finite set of *-polynomials with zero constant terms. By $C^{*}\langle G \mid \mathcal{R}\rangle$, we denote the universal (not-necessarily-unital) $C^{*}$-algebra generated by $g_{1}, \ldots, g_{l}$ subject to

$$
\left\|g_{j}\right\| \leq 1 \quad \text { and } \quad p_{i}\left(g_{1}, \ldots, g_{l}\right)=0
$$


By $C_{\epsilon}^{*}\langle G \mid \mathcal{R}\rangle$, we denote the universal unital $C^{*}$-algebra generated by $g_{1}, \ldots, g_{l}$ subject to

$$
\left\|g_{j}\right\| \leq 1+\epsilon \text { and }\left\|p_{i}\left(g_{1}, \ldots, g_{l}\right)\right\| \leq \epsilon .
$$

Sometimes, to be more explicit, we will denote the generators of $C_{\epsilon}^{*}\langle G \mid \mathcal{R}\rangle$ by $g_{1}^{\epsilon}, \ldots, g_{l}^{\epsilon}$. We let $P_{\epsilon}$ denote the surjection

$$
P_{\epsilon}: C_{\epsilon}^{*}\langle G \mid \mathcal{R}\rangle \rightarrow C^{*}\langle G \mid R\rangle
$$

which sends $g_{j}^{\epsilon}$ to $g_{j}$.

If, for every $\eta>0$, there exists $\epsilon>0$ and a $*$-homomorphism

$$
\sigma_{\epsilon}: C^{*}\langle G \mid \mathcal{R}\rangle \rightarrow C_{\epsilon}^{*}\langle G \mid \mathcal{R}\rangle
$$

such that

$$
\left\|\sigma_{\epsilon}\left(g_{j}\right)-g_{j}^{\epsilon}\right\| \leq \eta, \quad j=1, \ldots, l
$$

and $P_{\epsilon} \circ \sigma_{\epsilon}=$ id, then $R$ is stable.

Theorem 2.5. For a finitely presented $C^{*}$-algebra $C^{*}\langle G \mid \mathcal{R}\rangle$, the following conditions are equivalent:

(1) $\mathcal{R}$ is stable.

(2) $C^{*}\langle G \mid R\rangle$ is semiprojective.

(3) $C^{*}\langle G \mid R\rangle$ is corona semiprojective.

Proof. The implication (1) $\Rightarrow(2)$ follows from [8, Theorem 3.2] while (2) $\Leftrightarrow$ (3) is a special case of Theorem 2.4. For $(2) \Rightarrow(1)$, applying semiprojectivity to the identity map immediately gives a map $\bar{\sigma}_{\bar{\epsilon}}: C^{*}\langle G \mid \mathcal{R}\rangle \rightarrow C_{\bar{\epsilon}}^{*}\langle G \mid \mathcal{R}\rangle$ with $P_{\bar{\epsilon}} \circ \bar{\sigma}_{\bar{\epsilon}}=$ id. Let $\sigma_{\epsilon}$ equal the composition of $\bar{\sigma}_{\bar{\epsilon}}$ with the natural surjection of $C_{\bar{\epsilon}}^{*}\langle G \mid \mathcal{R}\rangle$ onto $C_{\epsilon}^{*}\langle G \mid \mathcal{R}\rangle$ for $\epsilon$ sufficiently small, $0<\epsilon<\bar{\epsilon}$.

\section{Generalizations of Kasparov's Technical Theorem.}

Using the techniques of [8] and [11] we derive several generalizations of Kasparov's Technical Theorem (KTT). Our goal is to find the closest possible thing to matrix units inside a corona algebra for $C^{*}$-subalgebras of the form $A \otimes F$ where $A$ is $\sigma$-unital and $F$ is finite-dimensional.

All our theorems involve a subset $D$ with which these ersatz matrix units are to commute. Easier proofs exist if one ignores $D$ and sticks with the separable case. Indeed, one may use the projectivity of $\mathrm{C} M_{n}$, or $\bigoplus C_{0}(0,1]$, and [12, Proposition 3.12.1] along the lines of an observation of Cuntz described in $[2, \S 12.4]$. We will discuss this further in recent joint work with Gert Pedersen [10]. 
In this section, $E$ will always denote a $\sigma$-unital $C^{*}$-algebra and $C(E)$ its corona algebra.

Theorem 3.1. Suppose $A_{1}, \ldots, A_{n}$ are $\sigma$-unital $C^{*}$-subalgebras of $C(E)$. Let $D$ be a separable, unital $C^{*}$-subalgebra of $C(E)$ such that

$$
A_{j} D A_{k}=0, \quad j \neq k .
$$

There exist $g_{1}, \ldots, g_{n}$ in $C(E) \cap D^{\prime}$ such that

$$
\begin{gathered}
0 \leq g_{j} \leq 1, \quad j=1, \ldots, n, \\
g_{j} g_{k}=0, \quad j \neq k, \\
g_{j} a=a g_{j}=a, \quad \forall a \in A .
\end{gathered}
$$

Proof. For $n=2$ this is equivalent to KTT. Indeed, it is very close to the equivalent result [11, Theorem 3.7]. An induction argument gives the general case.

Notice that $A_{1} A_{2}=0$ implies that the $C^{*}$-algebra generated by $A_{1} \cup A_{2}$ is isomorphic to $A_{1} \oplus A_{2}$. Therefore, Kasparov's Technical Theorem implicitly involves a $*$-homomorphism $A_{1} \oplus A_{2} \rightarrow C(E)$. A natural setting for generalization is $M_{n}(A) \rightarrow C(E)$.

Theorem 3.2. Suppose $A$ is a $\sigma$-unital $C^{*}$-algebra, $\varphi$ is a $*$-homomorphism

$$
\varphi: M_{n}(A) \rightarrow C(E)
$$

and $\operatorname{im}(\varphi)$ commutes with a separable subset $D$ of $C(E)$. There exists a*homomorphism

$$
\psi: \mathrm{C} M_{n} \rightarrow C(E) \cap D^{\prime}
$$

such that, setting $q_{i j}=\psi\left(t \otimes e_{i j}\right)$,

$$
q_{i j} \varphi\left(a \otimes e_{k l}\right)=\delta_{j k} \varphi\left(a \otimes e_{i l}\right), \quad \forall a \in A .
$$

Proof. Without loss of generality, $D$ may be assumed to be a unital $C^{*}$ algebra. Applying Theorem 3.1 to

$$
D, \varphi\left(A \otimes e_{11}\right), \ldots, \varphi\left(A \otimes e_{n n}\right)
$$

we obtain $g_{1}, \ldots, g_{n}$ in $C(E) \cap D^{\prime}$ such that

$$
0 \leq g_{i} \leq 1, \quad g_{i} g_{j}=0(i \neq j)
$$




$$
g_{j} \varphi\left(a \otimes e_{\jmath \jmath}\right)=\varphi\left(a \otimes e_{j j}\right)
$$

Let $h$ be a completely positive element of $A$. Since, for any $a$ in $A$,

$$
\begin{gathered}
g_{i} \varphi\left(h a h \otimes e_{j k}\right)=g_{i} g_{j} \varphi\left(h \otimes e_{j j}\right) \varphi\left(a h \otimes e_{j k}\right) \\
=\delta_{i j} \varphi\left(h a h \otimes e_{j k}\right)
\end{gathered}
$$

we conclude

$$
g_{i} \varphi\left(a \otimes e_{\jmath k}\right)=\delta_{\imath j} \varphi\left(a \otimes e_{j k}\right)
$$

for all $i, j, k$ and all $a \in A$.

Let $x=\varphi(h \otimes w)$ where

$$
w=\left[\begin{array}{llllll}
0 & & & & 1 \\
1 & 0 & & & \\
& 1 & \ddots & & \\
& & \ddots & 0 & \\
& & & 1 & 0
\end{array}\right] .
$$

Since $x$ is normal and both $x$ and $|x|=\varphi(h \otimes I)$ commute with $D$, we may apply [11, Theorem 4.4]. Thus, there exists $u$ in $C(E) \cap D^{\prime}$, with $\|u\| \leq 1$, such that $x=u|x|$ and $x^{*}=u^{*}|x|$.

Multiplying $x=u|x|$ by $\varphi\left(a h \otimes e_{i j}\right)$ yields

$$
u \varphi\left(h a h \otimes e_{i j}\right)=\varphi\left(h a h \otimes e_{i+1, j}\right) .
$$

(Addition taken $\bmod n$.) Therefore, by this and a similar calculation based on $x^{*}=u^{*}|x|$,

$$
u \varphi\left(a \otimes e_{i j}\right)=\varphi\left(a \otimes e_{i+1, j}\right) \quad \text { and } \quad u^{*} \varphi\left(a \otimes e_{i j}\right)=\varphi\left(a \otimes e_{\imath-1, j}\right),
$$

for all $j, k$ and all $a \in A$.

We now make a first approximation on what shall be the images, under $\psi$, of the generators $t \otimes e_{j 1}$ of $\mathrm{C} M_{n}$. Let

$$
a_{n}=g_{n} u^{n-1} g_{1},
$$

and then for $j=n-1, \ldots, 2$,

$$
a_{j-1}=g_{j-1} u^{j-2}\left|a_{j}\right| .
$$

Clearly $a_{\jmath} \in D^{\prime}$ and

$$
\left|a_{2}\right| \leq\left|a_{3}\right| \leq \cdots \leq\left|a_{n}\right| \leq 1 .
$$


By induction, $a_{j} \in \overline{g_{j} C(E) g_{1}}$. This forces some of the relations determining $\mathrm{C} M_{n}$ (as in [8, Proposition 2.7]) to hold, namely

$$
a_{j} a_{k}=0, \quad j, k=2, \ldots, n,
$$

$$
a_{j}^{*} a_{k}=0, \quad j \neq k
$$

We claim that, for all $b \in A$ and all $i, j, k$,

$$
a_{i} \varphi\left(b \otimes e_{j k}\right)=\delta_{1 j} \varphi\left(b \otimes e_{i k}\right) \text { and } a_{i}^{*} \varphi\left(b \otimes e_{j k}\right)=\delta_{i j} \varphi\left(b \otimes e_{1 k}\right) .
$$

For $i=n$ this follows directly from (1) and (2). But then

$$
\left|a_{n}\right| \varphi\left(b \otimes e_{j k}\right)=\delta_{1 j} \varphi\left(b \otimes e_{j k}\right)
$$

so one may handle the case $i=n-1$, et cetera.

As done in the proof of $[8$, Lemma 4.8], for $j=2, \ldots, n$ we define

$$
\tilde{a}_{j}=\lim _{m \rightarrow \infty} a_{j}\left((1 / m)+a_{j}^{*} a_{j}\right)^{-1 / 2}\left(a_{2}^{*} a_{2}\right)^{1 / 2} .
$$

By the calculations done in the proof of [8, Lemma 4.8] we conclude that setting $\psi\left(t \otimes e_{i 1}\right)=\tilde{a}_{i}$ defines a homomorphism

$$
\psi: \mathrm{C} M_{n} \rightarrow C(E) \cap D^{\prime}
$$

For every $b \in A,(5)$ implies

$$
\tilde{a}_{i} \varphi\left(b \otimes e_{j k}\right)=\delta_{1 j} \varphi\left(b \otimes e_{i k}\right) \text { and } \tilde{a}_{i}^{*} \varphi\left(b \otimes e_{j k}\right)=\delta_{i j} \varphi\left(b \otimes e_{1 k}\right)
$$

whence

$$
\psi\left(t \otimes e_{i j}\right) \varphi\left(b \otimes e_{k l}\right)=\delta_{j k} \varphi\left(b \otimes e_{i l}\right)
$$

\section{Interval stretching in corona algebras.}

We continue in this section to assume $C(E)$ is the corona algebra of some $\sigma$-unital $C^{*}$-algebra.

Let us consider a simple case of Kasparov's Technical Theorem. Given $h_{1}, h_{2}$ in $C(E)$ such that

$$
0 \leq h_{i} \leq 1(i=1,2) \quad \text { and } \quad h_{1} h_{2}=0
$$


the conclusion is there exists an additional element so that now

$$
0 \leq z \leq 1,0 \leq h_{i} \leq 1(i=1,2)
$$

$$
h_{1} z=0, h_{2} z=h_{2} \text { and } \quad h_{1} h_{2}=0 .
$$

The universal $C^{*}$-algebra for these relations are as follows:

$$
\left.C^{*}\left\langle h_{1}, h_{2}\right|(7) \text { holds }\right\rangle \cong C_{0}([-1,0) \cup(0,1])
$$

and

$$
\left.C^{*}\left\langle h_{1}, h_{2}, z\right|(8) \text { holds }\right\rangle \cong C_{0}([-1,0) \cup(0,2]) .
$$

For this reason, we think of Kasparov's Technical Theorem as a device for stretching an interval algebra at a point.

We introduce some notation to be used for the rest of this section.

Let $X \subseteq \mathbb{C}$ denote the union of the unit circle and the interval $[-2,-1]$. Let

$$
A_{n}=\left\{f \in C\left(X, M_{n}\right) \mid f(-2) \text { is scalar }\right\}
$$

and let $\alpha: M_{n}\left(C_{0}(0,1)\right)^{\sim} \rightarrow A_{n}$ denote the inclusion of the subalgebra of functions in $C\left(X, M_{n}\right)$ that are constant and scalar on $[-2,-1]$.

Lemma 4.1. Let $B$ denote any separable, unital $C^{*}$-algebra. Given a*homomorphism

$$
\varphi: M_{n}\left(C_{0}(0,1)\right)^{\sim} \otimes B \rightarrow C(E)
$$

whose image commutes with a separable subset $D \subseteq C(E)$, there exists *homomorphism

$$
\tilde{\varphi}: A_{n} \otimes B \rightarrow C(E)
$$

such that $\tilde{\varphi} \circ\left(\alpha \otimes \mathrm{id}_{B}\right)=\varphi$ and whose image commutes with $D$.

Proof. Since $A_{n}$ and $M_{n}\left(C_{0}(0,1)\right)^{\sim}$ are nuclear there is no ambiguity in the tensor product. As the tensor products involve unital $C^{*}$-algebras they are characterized as the universal $C^{*}$-algebras containing commuting copies of the two factors. By altering the subset $D$ one easily shows that it suffices to prove this result only when $B=\mathbb{C}$.

Proposition 2.8 of [8] shows that $M_{n}\left(C_{0}(0,1)\right)^{\sim}$ is the universal unital $C^{*}$-algebra generated by $x, a_{2}, a_{3}, \ldots, a_{n}$ subject to the relations

$$
\begin{gathered}
\left\|a_{j}\right\| \leq 1, \quad j=2, \ldots, n, \\
a_{j} a_{k}=0, \quad 2 \leq j, k \leq n \\
a_{j}^{*} a_{k}=0, \quad j \neq k,
\end{gathered}
$$




$$
\begin{gathered}
a_{j}^{*} a_{j}=x^{*} x, \\
x^{*} x=x x^{*}=-x-x^{*} .
\end{gathered}
$$

Similarly, one may show that $A_{n}$ is the universal unital $C^{*}$-algebra generated by $x, b_{2}, b_{3}, \ldots, b_{n}$ subject to the relations

$$
\begin{gathered}
\left\|b_{j}\right\| \leq 1, \quad j=2, \ldots, n, \\
b_{j} b_{k}=0, \quad 2 \leq j, k \leq n, \\
b_{j}^{*} b_{k}=0, \quad j \neq k, \\
b_{j}^{*} b_{j}=b_{k}^{*} b_{k}, \quad 2 \leq j, k \leq n, \\
\left(b_{j}^{*} b_{j}-1\right)\left(x x^{*}+x^{*} x\right)=0, \\
x x^{*}=x^{*} x=-x-x^{*},
\end{gathered}
$$

and the inclusion $\alpha$ corresponds to the $*$-homomorphism determined by the assignment $x \mapsto x, a_{j} \mapsto b_{j}|x|$. Working with the same relations, but in nonunital category, one sees that this is a special case of Theorem 3.2.

Lemma 4.2. Suppose $J$ is an ideal in $A$ and $A$ is a sub-C ${ }^{*}$-algebra of $B$. Let $J_{B}$ denote the ideal of $B$ generated by $J$. There is an isomorphism

$$
\Phi: B / J_{B} \rightarrow B *_{A}(A / J)
$$

defined by $\Phi\left(b+J_{B}\right)=b$.

We will need to prove technical results regarding maps from general dimension-drop graphs into corona algebras. For clarity we will concentrate on the most important case, that of the dimension-drop intervals, $\tilde{\mathbb{I}}_{n}$. Recall

$$
\tilde{\mathbb{I}}_{n}=\{f \in C[0,1] \mid f(0), f(1) \in \mathbb{C} I\},
$$

this being the unital version of the dimension-drop interval.

Although isomorphic to $\tilde{\mathbb{I}}_{n}$ we also consider

$$
\mathbb{J}_{n}=\{f \in C[-1,2] \mid f(-1) \text { and } f(2) \text { are scalar }\} .
$$

Let $\iota: \tilde{\mathbb{I}}_{n} \rightarrow \mathbb{J}_{n}$ denote the inclusion that extends a function to be constant on $[-1,0]$ and on $[1,2]$.

Theorem 4.3. Suppose $\varphi: \tilde{\mathbb{I}}_{n} \rightarrow C(E)$ is a*-homomorphism whose image commutes with a separable subset $D$. Then there exists a *-homomorphism $\bar{\varphi}: \mathbb{J}_{n} \rightarrow C(E) \cap D^{\prime}$ such that $\bar{\varphi} \circ \iota=\varphi$. 
Proof. Consider $M_{n}\left(C_{0}(0,1)\right)^{\sim} \otimes C[0,1]$ which we identify with

$$
C_{n}=\left\{f \in C\left([0,1]^{2}, M_{n}\right) \mid f(0, t)=f(1, t) \in \mathbb{C} I, \forall t\right\} .
$$

Restriction to the diagonal gives us a surjection

$$
\rho: M_{n}\left(C_{0}(0,1)\right)^{\sim} \otimes C[0,1] \rightarrow \tilde{\mathbb{I}}_{n} .
$$

One can check that by the last lemma we have the commutative diagram

$$
\begin{aligned}
& \left(A_{n} \otimes C[0,1]\right) *_{C_{n}} \tilde{\mathbb{I}}_{n} \stackrel{\cong}{\longrightarrow} \mathbb{J}_{n} \\
& \uparrow(\alpha \otimes \text { id }) * \text { id } \uparrow 。 \\
& C_{n} *_{C_{n}} \tilde{\mathbb{I}}_{n} \stackrel{\cong}{\longrightarrow} \tilde{\mathbb{I}}_{n}
\end{aligned}
$$

and so this result thus follows from Lemma 4.1.

Remark. The generalization of Theorem 4.3 to the case of extending maps of dimension-drop graphs into corona algebras follows by the same methods, but the notation is significantly worse.

\section{Stability for dimension-drop graphs.}

Suppose $X$ is a graph. We denote the associated dimension-drop $C^{*}$-algebra by

$$
C_{\text {vert }}\left(X, M_{n}\right)=\left\{f \in C\left(X, M_{n}\right) \mid f(v) \in \mathbb{C} I \text { for all vertices } v\right\}
$$

Theorem 5.1. For every graph $X$, and every positive integer $n$, the $C^{*}$ algebra $C_{\text {vert }}\left(X, M_{n}\right)$ is universal for a stable set of relations.

Proof. We may reduce to the case of $X$ connected using Proposition 3.10 and [8, Theorem 5.1]. For connected graphs, the proof is by induction on the number of vertices. If there is but one vertex then

$$
C_{\text {vert }}\left(X, M_{n}\right) \cong\left(\bigoplus_{j=1}^{J} M_{n}\left(C_{0}(0,1)\right)\right)^{\sim}
$$

where $J$ is the number of edges. This has stable relations by [8, Theorem 5.1].

Now suppose $X$ has at least two vertices, $v_{0}$ and $v_{1}$. We will need an auxiliary space, $\tilde{X}$, which is obtained from $X$ by stretching all edges attached 
to $v_{0}$ or $v_{1}$. Topologically, $\tilde{X}$ will be a copy of $X$. We shall use $v_{0}$ and $v_{1}$ to denote the appropriate vertices in $\tilde{X}$.

Choose a function

$$
h_{0}: \tilde{X} \rightarrow[-1,2]
$$

such that $h_{0}^{-1}([-1,0])$ consists of the union of half-closed subintervals, containing $v_{0}$, of each edge adjacent to $v_{0}$. We may assume a similar statement holds for $h_{0}^{-1}([1,2])$ and $v_{1}$.

We will identify $X$ with the quotient of $\tilde{X}$ obtained by collapsing $h_{0}^{-1}([-1,0])$ to a point and $h_{0}^{-1}([1,2])$ to a different point. We will also consider two copies of the graph obtained from $X$ by collapsing the two designated vertices together. We let $\tilde{Y}$ denote the quotient of $\tilde{X}$ obtained by identifying $v_{0}$ with $v_{1}$ and $Y$ denote the quotient of $\tilde{X}$ obtained by collapsing $h_{0}^{-1}([-1,0]) \cup h_{0}^{-1}([1,2])$ to a point.

Accordingly, we will be making identifications of the various dimensiondrop algebras with subalgebras of $C\left(\tilde{X}, M_{n}\right)$. Of course, $C_{\text {vert }}\left(\tilde{X}, M_{n}\right)$ is defined as such a subalgebra. The remaining identifications are:

$$
\begin{aligned}
C_{\text {vert }}\left(X, M_{n}\right)=\left\{f \mid f(x)=f\left(v_{0}\right) \text { if } h_{0}(x) \leq 0\right. \\
\left.\quad \text { and } f(x)=f\left(v_{1}\right) \text { if } h_{0}(x) \geq 1\right\}, \\
C_{\text {vert }}\left(Y, M_{n}\right)=\left\{f \mid f(x)=f\left(v_{0}\right) \text { if } h_{0}(x) \leq 0 \text { or } h_{0}(x) \geq 1\right\} \\
C_{\text {vert }}\left(\tilde{Y}, M_{n}\right)=\left\{f \mid f\left(v_{0}\right)=f\left(v_{1}\right)\right\} .
\end{aligned}
$$

Our strategy is based on the observation that $C_{\text {vert }}\left(X, M_{n}\right)$ is generated by the subalgebra $C_{\text {vert }}\left(Y, M_{n}\right)$ and the element

$$
h=h_{1} \otimes I \quad \text { where } \quad h_{1}(x)=\max \left(\min \left(h_{0}(x), 1\right), 0\right) .
$$

A way to express the relation between $h$ and $C_{\text {vert }}\left(Y, M_{n}\right)$ is that

$$
e^{2 \pi i h}=e^{2 \pi i h_{1}} \otimes I .
$$

By Theorem 2.6, our task is reduced to proving corona semiprojectivity for $C_{\text {vert }}\left(X, M_{n}\right)$ while assuming it for $C_{\text {vert }}\left(\tilde{Y}, M_{n}\right)$. So suppose that we are given a unital $*$-homomorphism

$$
\varphi: C_{\text {vert }}\left(X, M_{n}\right) \rightarrow C(E) \cong B / \widetilde{\bigcup I_{m}}
$$

By Theorem 4.3 and the remark following, there is an extension of $\varphi$ to

$$
\bar{\varphi}: C_{\text {vert }}\left(\tilde{X}, M_{n}\right) \rightarrow C(E) .
$$

By the induction hypothesis, the restriction of $\bar{\varphi}$ to $C_{\text {vert }}\left(\tilde{Y}, M_{n}\right)$ can be lifted to

$$
\psi: C_{\mathrm{vert}}\left(\tilde{Y}, M_{n}\right) \rightarrow B / I_{m}
$$


for some $m$. This leads to the following commutative diagram:

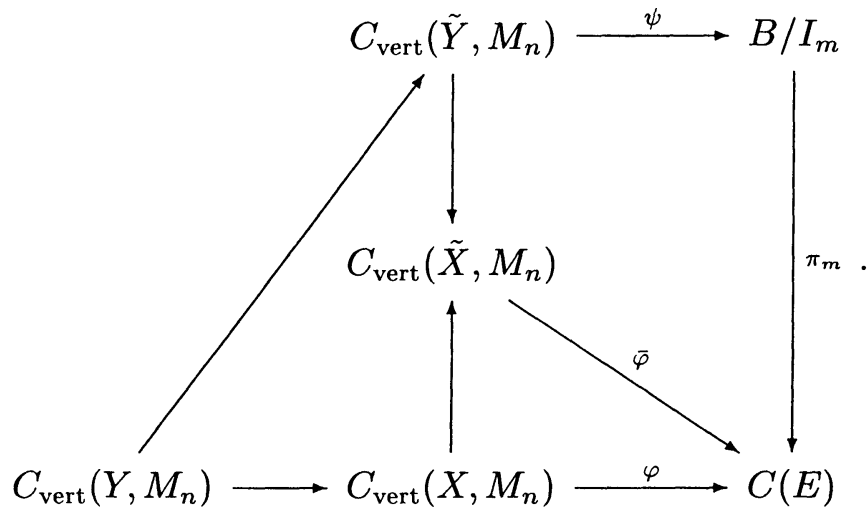

Let $H$ be any lift of $\varphi(h)$ to $B / I_{m}$ such that $0 \leq H \leq 1$. Now define

$$
\tilde{H}=\psi\left(l\left(h_{0}\right) \otimes I\right)+\psi\left(m\left(h_{0}\right)^{1 / 2} \otimes I\right) H \psi\left(m\left(h_{0}\right)^{1 / 2} \otimes I\right)
$$

where $l$ and $m$ are the functions

$$
l(t)=\left\{\begin{array}{cc}
0, & t \leq 0, \\
t, & 0 \leq t \leq 1, \\
2-t, & 1 \leq t \leq 2,
\end{array} \quad m(t)=\left\{\begin{array}{cc}
-t, & t \leq 0 \\
0, & 0 \leq t \leq 1 \\
t-1, & 1 \leq t \leq 2
\end{array}\right.\right.
$$

These are defined so that $l+m h_{2}=h_{2}$ where $h_{2}$ is the function

$$
h_{2}(t)=\left\{\begin{array}{l}
0, \quad t \leq 0 \\
t, 0 \leq t \leq 1 \\
1,1 \leq t \leq 2
\end{array}\right.
$$

Notice also that $h_{2}\left(h_{0}\right)=h_{1}$.

Clearly $\tilde{H}$ is selfadjoint. In fact, it is also a lift of $\varphi(h)$ since

$$
\begin{aligned}
\pi_{m}(\tilde{H}) & =\bar{\varphi}\left(l\left(h_{0}\right) \otimes I\right)+\bar{\varphi}\left(m\left(h_{0}\right) \otimes I\right) \bar{\varphi}\left(h_{2}\left(h_{0}\right) \otimes I\right) \\
& =\bar{\varphi}\left(\left(l+m h_{2}\right)\left(h_{0}\right) \otimes I\right)=\varphi(h) .
\end{aligned}
$$

For any $f \otimes T \in C_{\text {vert }}\left(Y, M_{n}\right)$

$$
(f \otimes T)\left(m\left(h_{0}\right)^{1 / 2} \otimes I\right)=0 \quad \Rightarrow \quad \psi(f \otimes T) \tilde{H}=\tilde{H} \psi(f \otimes T) .
$$

By replacing $\tilde{H}$ by $h_{2}(\tilde{H})$, we have found a lift of $\varphi(h)$, with $0 \leq \tilde{H} \leq 1$, and a lift of $\left.\varphi\right|_{C_{\text {vert }}\left(Y, M_{n}\right)}$ that commute.

Expressing this conclusion differently, we have shown that given a unital $\operatorname{map}$

$$
C_{\text {vert }}\left(X, M_{n}\right) \rightarrow C(E)
$$


we can find an $m$ and a map making the diagram commute where $D$ is the universal unital $C^{*}$-algebra generated by a copy of $C_{\text {vert }}\left(Y, M_{n}\right)$ and a central element $h$ such that $0 \leq h \leq 1$. I.e.,

$$
D \cong C_{\text {vert }}\left(Y, M_{n}\right) \otimes C[0,1] .
$$

We have no further need for $\tilde{X}$ so $v_{0}$ and $v_{1}$ again denote the specified vertices in $X$. We regard $Y$ as the quotient of $X$, with quotient map $\eta: X \rightarrow$ $Y$ which collapses $v_{0}$ and $v_{1}$ to a single vertex we call $w_{0}$.

Let us identify $D$ with

$$
\left\{g \in C\left(Y \times[0,1], M_{n}\right) \mid g(v, t) \in \mathbb{C} I \text { for all vertices }\right\} .
$$

The copy of $C_{\text {vert }}\left(Y, M_{n}\right)$ and the extra element $h$ appear as functions in $D$ constant in one variable or the other. There is a sort of diagonal map

$$
\Delta: X \rightarrow Y \times[0,1], \quad \Delta(x)=\left(\eta(x), h_{1}(x)\right)
$$

which induces a surjection $\beta: D \rightarrow C_{\text {vert }}\left(X, M_{n}\right)$.

We need also a quotient of $D$ where the relation (9) holds approximately. Consider

$$
Z_{\delta}=\left\{(\eta(x), t) \in Y \times[0,1]|| e^{2 \pi i h_{1}(x)}-e^{2 \pi i t} \mid \leq \delta\right\},
$$

where $\delta$ is a small number to be named later, and let

$$
D_{\delta}=\left\{g \in C\left(Z, M_{n}\right) \mid g(v, t) \in \mathbb{C} I \text { for all vertices }\right\} .
$$

Since $\Delta$ maps into $Z$ it induces

$$
\beta_{0}: D_{\delta} \rightarrow C_{\text {vert }}\left(X, M_{n}\right) .
$$

By increasing $m$ we may assume that the map $D \rightarrow B / I_{m}$ factors through $D_{\delta}$. Therefore, we are done if we exhibit a right-inverse to $\beta_{0}$. This exists because there is a retraction of $Z_{\delta}$ onto $\operatorname{im}(\Delta)$ which sends $(v, t)$ to $\left(v, t^{\prime}\right)$ for every vertex $v$. To be able to describe this retraction we break up $Z_{\delta}$ as $Z_{\delta}=Z_{1} \cup Z_{2} \cup Z_{3}$ where

$$
\begin{aligned}
& Z_{1}=\left\{(\eta(x), t)|| h_{1}(x)-t \mid \leq 1 / 4,0<t<1\right\} \\
& Z_{2}=\left\{(\eta(x), t)|| h_{1}(x)+1-t \mid \leq 1 / 4\right\} \\
& Z_{3}=\left\{(\eta(x), t)|| h_{1}(x)-1-t \mid \leq 1 / 4\right\} .
\end{aligned}
$$

The retraction sends $Z_{2}$ to $\left(w_{0}, 1\right)$ and $Z_{3}$ to $\left(w_{0}, 0\right)$. Each point $(\eta(x), t)$ in $Z_{1}$ is sent to $(\eta(x), s)$ where $s$ is the unique number in $(0,1)$ such that 
$e^{2 \pi i s}=e^{2 \pi i h_{1}(x)}$. By choosing $\delta$ sufficiently small, we ensure that $(v, t) \notin$ $Z_{2} \cup Z_{3}$ for any vertex $v$ except for $v=w_{0}$. Therefore this is the desired retraction.

\section{References}

[1] B. Blackadar, Shape theory for $C^{*}$-algebras, Math. Scand., 56 (1985), 249-275.

[2] — K-theory for Operator Algebras, M.S.R.I. Monographs No. 5, Springer Verlag, Berlin and New York, 1986.

[3] L. Brown, Unitary equivalence modulo the compact operators and extensions of $C^{*}$ algebras, Proceedings of a Conference on Operator Theory, Lecture Notes in Mathematics, vol. 345, Springer-Verlag, Berlin, 1973, 58-140.

[4] M. Dadarlat and T.A. Loring, $K$-homology, asymptotic morphisms and unsuspended E-theory, J. Funct. Anal., 126 (1994), 367-383.

[5] — Extensions of certain real rank zero $C^{*}$-algebras, Ann. Inst. Fourier (Grenoble), 44 (1994), 906-925.

[6] E. Effros and J. Kaminker, Homotopy continuity and shape theory for $C^{*}$-algebras, Geometric Methods in Operator Algebras, U.S. - Japan Joint Seminar at Kyoto, 1983, Pitman.

[7] G.A. Elliott, On the classification of $C^{*}$-algebras of the real rank zero, J. reine angew Math., 443 (1993), 179-219.

[8] T.A. Loring, $C^{*}$-algebras generated by stable relations, J. Funct. Anal., 112 (1993), 159-201.

[9] _ Projective $C^{*}$-algebras, Math. Scan., 173 (1993), 274-280.

[10] T.A. Loring and G.K. Pedersen, Projectivity, transitivity and AF telescopes, preprint.

[11] C.L. Olsen and G.K. Pedersen, Corona $C^{*}$-algebras and their applications to lifting problems, Math. Scand., 164 (1989), 63-86.

[12] G.K. Pedersen, $C^{*}$-algebras and Their Automorphism Groups, Academic Press, New York, 1979.

Received September 3, 1993 and revised April 15, 1995. This work was partially supported by NSF grant DMS-900734 and NATO grant CRG-920777.

UNIVERSiTy of New Mexico

ALBUQUeRque NM 87131

E-mail address: loring@math.unm.edu 

Rosa M. Miró-Roig, Singular moduli spaces of stable vector bundles on $\mathbf{P}^{3}$

Hitoshi Moriyoshi and Toshikazu Natsume, The Godbillon-Vey Cyclic Cocycle and Longitudinal Dirac Operators

J.C. Naranjo, The positive dimensional fibres of the Prym map

Artur Nicolau and Arne Stray, Nevanlinna's coefficients and Douglas al-

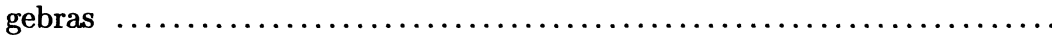

K.K. Park, Entropy of a skew product with a $Z^{2}$-action $\ldots \ldots \ldots \ldots \ldots .227$

María Cristina Pereyra, Sobolev spaces on Lipschitz curves ......... 553

T. Sano, Commuting co-commuting squares and finite dimensional Kac algebras

H.B. Thompson, Second order ordinary differential equations with fully nonlinear two point boundary conditions

H.B. Thompson, Second order ordinary differential equations with fully nonlinear two point boundary conditions II

F. $\mathrm{Xu}$, The flat part of non-flat orbifolds

Hidenobu Yoshida, A type of uniqueness for the Dirichlet problem on a half-space with continuous data 


\section{PACIFIC JOURNAL OF MATHEMATICS}

Volume $172 \quad$ No. $2 \quad$ February 1996

On the failure cycles for the quadratic normality of a projective variety

307

EdoARdo BaLlico

On the minimal free resolution of general embeddings of curves

315

EdOARDO BALlico

On normality of the closure of a generic torus orbit in $G / P$

321

ROMUALD DABROWSKI

Paragroupe d'Adrian Ocneanu et algèbre de Kac

331

MARIE-Claude DAVID

Irreducibility and dimension theorems for families of height 3 Gorenstein algebras

SuSAN J. DiESEL

On the cohomology of the Lie algebra $L_{2}$

ALICE FIALOWSKI

Generic differentiability of convex functions on the dual of a Banach space

John R. Giles, P. S. Kenderov, WarRen Brian Moors and S. D.

SCIFFER

Moon hypersurfaces and some related existence results of capillary hypersurfaces

without gravity and of rotational symmetry

FEI-TSEN LIANG

Stable relations. II. Corona semiprojectivity and dimension-drop $C^{*}$-algebras

TERRY ATHERTON LORING

Singular moduli spaces of stable vector bundles on $\mathbf{P}^{3}$

Rosa M. MIRó-ROIG

The Godbillon-Vey cyclic cocycle and longitudinal Dirac operators

Hitoshi MoRIYOSHI and TOSHIKAZU NATSUME

Nevanlinna's coefficients and Douglas algebras

ARTUR NiCOLAU and ARNE STRAY

Sobolev spaces on Lipschitz curves

MARÍA CRISTINA PEREYRA

A type of uniqueness for the Dirichlet problem on a half-space with continuous data 